\title{
The Ritvo Autism Asperger Diagnostic Scale-Revised (RAADS-R): A Scale to Assist the Diagnosis of Autism Spectrum Disorder in Adults: An International Validation Study
}

\author{
Riva Ariella Ritvo • Edward R. Ritvo • Donald Guthrie • \\ Max J. Ritvo • Demetra H. Hufnagel • William McMahon • \\ Bruce Tonge $\cdot$ David Mataix-Cols $\cdot$ Amita Jassi $\cdot$ \\ Tony Attwood · Johann Eloff
}

Published online: 18 November 2010

(C) The Author(s) 2010. This article is published with open access at Springerlink.com

\begin{abstract}
The Ritvo Autism Asperger Diagnostic ScaleRevised (RAADS-R) is a valid and reliable instrument to assist the diagnosis of adults with Autism Spectrum Disorders (ASD). The 80-question scale was administered to 779 subjects (201 ASD and 578 comparisons). All ASD subjects met inclusion criteria: DSM-IV-TR, ADI/ADOS diagnoses and standardized IQ testing. Mean scores for each of the questions and total mean ASD vs. the comparison groups' scores were significantly different $(p<.0001)$. Concurrent validity with Constantino Social Responsiveness Scale-Adult $=95.59 \%$. Sensitivity $=97 \%$, specificity $=100 \%$, test-retest reliability $r=.987$. Cronbach
\end{abstract}

\author{
R. A. Ritvo \\ The Child Study Center, Yale University School of Medicine, \\ New Haven, CT, USA \\ E. R. Ritvo - D. Guthrie \\ The Geffen School of Medicine at UCLA, Los Angeles, CA, \\ USA \\ M. J. Ritvo - D. H. Hufnagel \\ Yale College, New Haven, CT, USA \\ W. McMahon \\ University of Utah School of Medicine, Salt Lake, UT, USA \\ B. Tonge \\ Monash University, Melbourne, VIC, Australia \\ D. Mataix-Cols · A. Jassi \\ King's College London, London, UK \\ T. Attwood · J. Eloff \\ Griffith University, Brisbane, QLD, Australia \\ R. A. Ritvo ( $\square)$ \\ 11941 Saltair Terrace, Los Angeles, CA 90049, USA \\ e-mail: ariella.ritvo@yale.edu
}

alpha coefficients for the subscales and 4 derived factors were good. We conclude that the RAADS-R is a useful adjunct diagnostic tool for adults with ASD.

Keywords Diagnosis · Autism · Asperger's disorder · RAADS-R

\section{Introduction}

The RAADS was designed to address a major gap in screening services for adults with autism spectrum disorders. With the increased prevalence of the condition and the fact that adults are being referred or self-referred for services or diagnosis with increasing frequency, this instrument is a useful clinical tool to assist clinicians with the diagnosis of this growing population of higher functioning individuals in adulthood. This study was designed to standardize the Ritvo Autism Asperger Diagnostic Scale-Revised (RAADS-R) in a large number of subjects at nine centers on three continents.

The RAADS- $\mathrm{R}$ is a modified version of the Ritvo Autism Asperger's Diagnostic Scale (Ritvo et al. 2008). The rationale for its development was the need for a clinical adjunct diagnostic tool. While several diagnostic scales for children and adolescents with ASD are available (Attwood 1998; Ehlers et al. 1999), only one peer- reviewed scale, The Autism-Spectrum Quotient (AQ) was designed specifically for adults (Baron-Cohen et al. 2001). Its authors emphasize that it is useful in identifying autistic traits and as a screening instrument but not as a diagnostic tool. The AQ is mailed in by the participant, unlike the RAADS-R, which is designed to be administered by a clinician in a clinical setting. Baron-Cohen et al. (2005) propose a diagnostic system as well: The Adult Asperger's 
Assessment (AAA): A Diagnostic Method. While the AAA is a good system, it is a complete diagnostic system, unlike the RAADS-R. It is lengthy and complicated to administer and is still relatively new. The initial RAADS was developed around the same time period. The original RAADS contains 78 empirically derived questions that assess symptoms based upon DSM-IV-TR (American Psychiatric Association 2000) and ICD-10 World Health Organization (1992) diagnostic criteria that were extensively tested prior to inclusion on the scale. It proved accurate in discriminating autistic and Asperger's subjects $(N=37)$ from comparison subjects $(N=57)$ when tested (e.g., sensitivity $=1$, specificity $=1$ ). Seventy-six of the 78 questions significantly distinguished ASD subjects from comparison subjects (Ritvo et al. 2008).

Questions on the initial RAADS assess developmental pathology in three symptom areas: language, social relatedness, and sensory-motor (re: DSM-IV-TR). After critical review and the results of a factor analysis, a revised 80item version was developed (the RAADS-R) with the addition of a fourth symptom area (circumscribed interests), two questions, and several word clarifications. Like the RAADS, the RAADS-R is a self-report instrument, designed for adults $(18+)$ with average or above average intelligence.

In order to validate the RAADS-R, a uniform research protocol was developed to be implemented at nine research centers on three continents. The protocol assured uniformity of diagnosis and data acquisition, and its implementation in multiple centers afforded an opportunity to assess the applicability of the scale in a variety of international clinical settings: (a) UCLA; (b) The Child Study Center, Yale University; (c) University of Utah; (d) Mt. Sinai Medical School, New York; (e) The Geneva Center, Toronto, Canada; (f) King's College, London, England; (g) Monash University, Melbourne, Australia; (h) Griffith University, Brisbane, Australia; and (i) ASPECT, Sidney, Australia.

At the time this research was concluding, the American Psychiatric Association's DSM-V committee proposed new diagnostic criteria for Autism Spectrum Disorders (APA DSM-5 2010). These new guidelines propose combining individuals who meet the DSM-IV-TR criteria for Autistic Disorder and Asperger's Disorder into one category called Autistic Spectrum Disorder (ASD). The results of this study will present subjects grouped according to both the DSM-IV-TR and proposed DSM-V criteria. There has been ongoing controversy among clinicians about the relationship of autism and Asperger's disorder. Diagnostic approaches and definitions are described in the literature (Fitzgerald and Corvin 2001; Klin et al. 2005; Volkmar and Lord 1998).

\section{Methods}

Participants

\section{Research Centers and Informed Consents}

Nine centers on three continents were selected to provide a worldwide spectrum of English speaking subjects. Human Subject Protection Protocol and Consent to Participate Forms were approved by the California Institutional Review Board, Pasadena, California for all UCLA subjects, and served as models for the other centers to assure uniformity. Each center has ongoing clinical and research programs focusing on autism, and each center complied with their rules and regulations concerning the protection of human subjects. Each of the nine centers provided autistic and Asperger's disorder subjects and some also contributed comparison subjects.

\section{Participant Grouping}

The study population included 4 groups as follows: (a) subjects with a diagnosis of Autistic Disorder $(n=66)$, (b) subjects with a diagnosis of Asperger's Disorder $(n=135)$, (c) subjects with no previous DSM-IV-TR diagnosis $(n=276)$, and (d) subjects with an axis I DSMIV-TR diagnosis other than Autistic Disorder, Asperger's Disorder, or PDD NOS $(n=302)$. Subjects in this group had the following diagnoses: Social Phobia, Schizophrenia, Major Depressive Disorder, Bipolar Disorder Type I and II, Obsessive Compulsive Disorder, Dysthymic Disorder, Generalized Anxiety Disorder, Post Traumatic Stress Disorder, Psychotic Disorder NOS, Anorexia Nervosa and Polysubstance Dependence. For some data analyses, the above groups were collapsed into two groups as follows: (a) Autism Spectrum Disorder (ASD) subjects $(N=201)$, and (b) all comparison subjects $(N=578)$. No participants from either group declined to participate.

\section{Inclusion Criteria}

A standardized research protocol was implemented at each of the nine centers to assure uniformity of diagnosis. All ASD subjects had to meet the following criteria: (a) a clinical DSM-IV-TR diagnosis of autistic or Asperger's disorder, (b) an ADI/ADOS or at least an ADOS module 4 scale to assure they met the cut off for ASD, and (c) a Full Scale IQ score of 80 or above. If prior formal IQ test results were not available, a WAIS or a WASI was administered. Constantino's Social Responsiveness Scale-Adult Research Version (SRS-A) was obtained if a suitable informant accompanied the subject, as it is a second party rating scale (a second party rating scale is administered to a person 
other than the subject who answers questions about the subject, such as a family member or a friend). The SRS was selected because it is easy to administer and is reported to have good psychometric properties: internal consistency 0.91-0.97, test-retest reliability $0.84-0.97$ and interrater reliability 0.74-0.95 (Bolte et al. 2008). The respondents completing the measure in this study were spouses, parents and siblings. A subset of subjects at UCLA was retested after an average of 1 year to determine test-retest reliability.

Two groups of comparison subjects were selected. The first consisted of 276 volunteers who did not have, and never had a DSM-IV-TR diagnosis. They were recruited from clinic staff members and students, and were individually administered the scale by a research staff member. The second group consisted of 302 volunteers recruited at an adult psychiatric out-patient clinic. They were formally diagnosed as having a DSM-IV-TR diagnosis other than ASD by an experienced board certified psychiatrist or a licensed psychologist who administered the scale individually to each. The majority of the comparison cases were recruited by the principal investigator and UCLA.

\section{Materials}

Design of the Revised Scale: The RAADS-R contains 64 symptom-based questions and 16 non-symptom based responses (see Appendix 1 for scale and Appendix 2 for scoring instructions). The questions were divided into four sub scales per DSM-IV-TR criteria as follows:

1. Social Relatednesss-total of 39 questions: $1,6,8,11$, $14,17,18,25,37,38,3,5,12,28,39,44,45,76,79$, $80,20,21,22,23,26,31,43,47,48,53,54,55,60,61$, $64,68,69,72,77$.

2. Circumscribed Interests - total of 14 questions: 9, 13, 24, 30, 32, 40, 41, 50, 52, 56, 63, 70, 75, 78.

3. Language-total of 7 questions: 2, 7, 27, 35, 58, 66, 15.

4. Sensory Motor-total of 20 questions: 10, 19, 4, 33, 34, $36,46,71,16,29,42,49,51,57,59,62,65,67,73,74$.

Procedures

\section{Administration of the RAADS-R to ASD Subjects}

Following initial screening, a clinician interviewed each subject to confirm the diagnostic information and IQ data, completed the ADI/ADOS or ADOS module 4, and then introduced the scale and explained how to answer the questions. The clinician remained with each subject as the questions were being answered to offer clarifications when asked, and to make sure answers were entered in the proper columns on the Likert scale. An SRS-A scale was obtained from cooperating accompanying adults when available.

\section{Administration of the RAADS-R to Comparison Subjects}

After initial diagnostic and IQ screening, all were given the RAADS-R to complete in the presence of a clinician. Each of the nine participating centers was asked to score, review, and mail in full packets of ASD subjects. Some of the participating centers contributed data to the comparison group subject pool.

\section{Data Analysis}

Data were coded by each center to preserve anonymity prior to being sent to UCLA. Data were then recoded and entered into an Excel spreadsheet and then transferred to SAS and MPlus for statistical analyses. Exploratory factor analysis was carried out using both orthogonal and oblique varimax rotation under maximum likelihood estimation. A scree plot of the eigenvalues was examined to determine the number of factors. A confirmatory factor analysis was carried out on the final factors using robust weighted least squares (WLSMV) estimation methods as the original items are ordinal. The factor loadings were compared across gender and ASD subgroups to assess constancy (differential item function analysis). Cronbach alpha was also computed to assess the internal consistency of the derived factors. Unadjusted means were compared using one-way analysis of variance and demographics adjusted means were compared using analysis of covariance methods. Associations between continuous variables were assessed using Pearson correlations. Sensitivity, specificity and accuracy of a given factor or score for distinguishing between ASD versus non ASD was computed using non-parametric ROC (receiver operator characteristic) methods. Unweighted accuracy is defined as the average of sensitivity and specificity. The optimal threshold (cut point) was defined as the value of the given factor or score that maximized accuracy.

\section{Results}

Subjects' Demographics

Table 1 contains the sex distribution and demographic characteristics of the four subject groups.

Diagnostic Accuracy (Sensitivity and Specificity)

The total RAADS scores ranged from 44 to 227 in the ASD subjects and from 0 to 65 in the comparison groups. By ROC curve analysis, we determined that the best threshold 
Table 1 Demographic characteristics of the study sample

\begin{tabular}{|c|c|c|c|c|c|c|c|c|c|c|c|}
\hline \multirow[t]{2}{*}{ Group } & \multirow[t]{2}{*}{$N$} & \multicolumn{2}{|c|}{ Males } & \multicolumn{2}{|c|}{ Females } & \multirow[t]{2}{*}{ Mean age } & \multirow[t]{2}{*}{ Married \% } & \multicolumn{3}{|c|}{ Highest grade $\%$} & \multirow[t]{2}{*}{ Mean IQ } \\
\hline & & $N$ & $\%$ & $N$ & $\%$ & & & High school & College & Graduate & \\
\hline Autistic disorder & 66 & 52 & 79.1 & 14 & 20.9 & 30.81 & 13.4 & 52.2 & 37.3 & 10.4 & 114 \\
\hline Asperger's disorder & 135 & 93 & 68.9 & 42 & 31.1 & 32.01 & 21.5 & 37.8 & 53.3 & 7.4 & 122 \\
\hline ASD & 201 & 145 & 72.1 & 56 & 27.9 & 31.45 & 18.9 & 45.0 & 45.3 & 8.9 & 119 \\
\hline No DSM-IV-TR & 276 & 114 & 41.3 & 162 & 58.7 & 41.51 & 39.5 & 37.7 & 46.0 & 16.3 & 116 \\
\hline Other DSM-IV-TR & 302 & 134 & 44.4 & 168 & 55.6 & 42.04 & 28.5 & 40.1 & 53.3 & 6 & 112 \\
\hline Comparisons & 578 & 248 & 42.9 & 330 & 57.1 & 41.78 & 33.7 & 38.9 & 49.65 & 11.15 & 114 \\
\hline Full sample & 779 & 394 & 44.4 & 386 & 49.5 & & 29.9 & 39.9 & 49.4 & 10.3 & 116.5 \\
\hline
\end{tabular}

for distinguishing between the two groups was a score of 65 . Using this value, all 578 comparison subjects were correctly predicted with no "false positives" $($ specificity $=100 \%)$.

Six ASD subjects from three of the nine centers scored below the threshold (non-ASD predicted range). These "false negatives" yielded a sensitivity of $97 \%$ (see Table 2).

Table 3 shows the sensitivity, specificity and accuracy for the original four subscale domains and the four factors as well as the total RAADS-R score. As can be seen, the total RAADS-R score has the highest accuracy.

\section{Group Comparisons}

One-way ANOVA comparisons across all groups, mean RAADS-R scores, and statistical characteristics of subject groups are contained in Table 4 . The means and statistical characteristics obtained after collapsing the four groups into two (all ASD subjects vs. all comparison subjects) are shown in Table 5. The mean RAADS-R scores of the ASD, the autistic, and the Asperger's disorder groups (Tables 3 and 4) were significantly different from the means of the comparison groups $(p<.0001)$. This was also true at each of the nine research centers. The original subscale domains, the factors and the total score are all significantly different in ASD versus non ASD.

An age and sex adjusted ANOVA of total RAADS-R scores and subscale scores, across diagnostic groups

Table 2 Characteristics of the only 6 ASD subjects (3\%) whose mean RAADS-R scores were below 65 (false negatives) (mean age $=19.9)$

\begin{tabular}{lllllll}
\hline Center & Diagnosis & Age & Sex & School & IQ & RAADS-R \\
\hline YALE & ASPERGER & 19 & M & HS & 120 & 52 \\
GRIFITH & ASPERGER & 23 & M & HS & 117 & 56 \\
ASPEC & AUTISTIC & 18 & M & HS & 116 & 58 \\
GRIFITH & ASPERGER & 19 & M & HS & 122 & 58 \\
YALE & ASPERGER & 20 & M & COL & 116 & 59 \\
YALE & ASPERGER & 19 & M & COL & 116 & 60 \\
\hline
\end{tabular}

showed group differences. Sex was not significant as a main effect $(p=.06)$ but sex-diagnosis was significant $(p=.0043)$. The effect of diagnosis slightly varied by sex, however, diagnosis was the strongest effect. Age was significant as a main effect $(p=.0035)$ and age-diagnosis effect was also significant $(p<.05)$. In ASD subjects, an increase in age was positively correlated with an increase

Table 3 Accuracy in distinguishing ASD $(N=201)$ from non-ASD $(N=578)$ (ROC analysis)

\begin{tabular}{lllll}
\hline Variable & Threshold $^{\mathrm{a}} \begin{array}{l}\text { Sensitivity } \\
\%\end{array}$ & $\begin{array}{l}\text { Specificity } \\
\%\end{array}$ & $\begin{array}{l}\text { Accuracy } \\
\%\end{array}$ \\
\hline $\begin{array}{l}\text { Original subscale Dom } \\
\text { Social construct }\end{array}$ & 31 & 96.0 & 96.2 & 96.1 \\
$\begin{array}{l}\text { Circumscribed } \\
\text { interest }\end{array}$ & 15 & 89.6 & 97.1 & 93.3 \\
$\begin{array}{l}\text { Sensory motor } \\
\text { Language }\end{array}$ & 16 & 85.1 & 95.2 & 90.2 \\
$\begin{array}{l}\text { Total score } \\
\begin{array}{l}\text { Factor 1-Social } \\
\text { related }\end{array}\end{array}$ & 0.13 & 78.7 & 93.8 & 86.2 \\
$\begin{array}{l}\text { Factor 2- } \\
\quad \text { Circumscribed int. }\end{array}$ & 0.34 & 86.6 & 95.5 & 91.1 \\
$\begin{array}{l}\text { Factor 3-Sensory } \\
\text { motor }\end{array}$ & 0.36 & 71.8 & 92.5 & 82.2 \\
$\begin{array}{l}\text { Factor 4-Social } \\
\text { anxiety }\end{array}$ & 0.17 & 76.7 & 74.2 & 75.5 \\
\hline
\end{tabular}

${ }^{a}$ Value that best separates ASD from non ASD in SD units

Table 4 One way ANOVA comparisons and statistical characteristics of participant groups

\begin{tabular}{lrrrrrl}
\hline Group autistics & $N$ & Mean & Min & Max & SEp & SD \\
\hline Autistics & 66 & 138.46 & 44 & 227 & 4.61 & 41.4 \\
Asperger & 135 & 131.53 & 52 & 225 & 3.24 & 35.73 \\
No diagnosis & 276 & 21.39 & 0 & 65 & 0.93 & 65 \\
Other diagnosis & 302 & 30.11 & 2 & 65 & 0.89 & 65
\end{tabular}

This ANOVA is for total RAADS-R score by group

$F=286, \mathrm{~d} f=3, p<.0001$ 
Table 5 Means and statistical characteristics for total score, ASD vs. comparison groups

\begin{tabular}{llllllll}
\hline Group & Mean & SEp & SD & Range & S-W & Skewness & Kurtosis \\
\hline Autistic spectrum $(n=201)$ & 133.83 & 2.66 & 37.74 & $44-227$ & 0.993 & 0.09 & -0.18 \\
Comparison controls $(n=578)$ & 25.95 & 0.67 & 16.04 & $0-65$ & 0.959 & 0.42 & -0.76 \\
\hline
\end{tabular}

$S-W$ Shapiro-Wilkes normality statistic

$F=1,522.44, \mathrm{~d} f=1, p<.0001$

in mean RAADS-R scores. (Spearman correlation 0.37, $p=.0003)$. However, age-sex-diagnosis effect was not significant $(p=.1299)$. Repeated analyses after adjustment for sex and age between and among the subject groups showed significant differences between ASD and comparison groups $(p<.0001)$.

\section{Pair-Wise Comparison of Each Question}

Post-hoc unequal variance $t$ tests were performed for pair-wise comparison of each of the 80 questions. The ASD subjects had significantly greater scores on each of the individual RAADS-R items $(N=80)$ than either of the comparison groups $(p<.0001$ for all comparisons). Total RAADS-R mean scores between autistic and Asperger's disorder subject groups were not significantly different.

\section{Validity Measures}

All ASD subjects met the research diagnostic criteria of each center and had an ADOS module 4 score in the autism range (these were inclusion criteria). SRS-A scales were administered to 69 first-degree relatives of ASD subjects. The concordance rate was $95.59 \%$ (all but three scored in the "autism" range).

\section{Analysis of the Subscales}

Mean scores and Cronbach alpha correlation coefficients were computed for each of the four subscale domains described previously: circumscribed interests $=.903$, language $=.789$, sensory motor $=.905$, social relatedness $=.923$ (for the four participant groups' mean scores of the subscales, see Table 6).

\section{Factor Analysis}

Factor Analysis was carried out using both varimax (orthogonal) and oblique (non-orthogonal) varimax rotation. The oblique rotational method was preferred because it assumes the underlying constructs to be interrelated. In addition, the oblique model had simpler factor structure that was easier to interpret.
Table 6 Statistical analysis of the subscales

\begin{tabular}{|c|c|c|c|}
\hline Group & Subscale & Mean & SD \\
\hline \multicolumn{4}{|c|}{ Autistic spectrum $(n=66)$} \\
\hline & Social & 67.89 & 21.45 \\
\hline & Sensory motor & 32.82 & 12.25 \\
\hline & Circumscribed interests & 28.11 & 8.54 \\
\hline & Language & 11.08 & 4.54 \\
\hline \multicolumn{4}{|c|}{ Asperger $(n=135)$} \\
\hline & Social & 65.07 & 17.80 \\
\hline & Sensory motor & 28.96 & 13.07 \\
\hline & Circumscribed interests & 27.44 & 8.76 \\
\hline & Language & 10.06 & 4.37 \\
\hline \multicolumn{4}{|c|}{ No DSM IV $(n=276)$} \\
\hline & Social & 9.24 & 7.70 \\
\hline & Sensory motor & 5.26 & 5.33 \\
\hline & Circumscribed interests & 5.03 & 4.44 \\
\hline & Language & 1.86 & 2.46 \\
\hline \multicolumn{4}{|c|}{ Other DSM IV $(n=302)$} \\
\hline & Social & 13.88 & 9.95 \\
\hline & Sensory motor & 7.72 & 4.92 \\
\hline & Circumscribed interests & 7.08 & 4.69 \\
\hline & Language & 1.43 & 1.76 \\
\hline
\end{tabular}

Four factors were identified using the oblique rotation method. These factors had a simpler factor structure with much less cross loading compared to the orthogonal results, making for easier interpretation. These final oblique factors also had a higher correlation with the original subscales compared to the orthogonal factors. Factors I and IV correlated most strongly with the social construct $(r=.80)$. Factor II correlated most strongly with circumscribed interests $(r=.78)$, factor III with sensory motor $(r=.88)$. The language construct was not picked up with the fourfactor model. The variables determined below are based on factor scores.

1. Factor I: Social Relatedness, containing questions related to empathy, intimacy and social language accounted for $23.9 \%$ of the variance. Questions: 1, 6, $8,11,13,14,15,16,18,31,37,38,43,48,52,53,58$, $62,66,68,72,74,77 ; \alpha=.93$. 
2. Factor II: Circumscribed Interests, also with questions relating to social blindness, accounted for $25.0 \%$ of the variance. Questions: 3, 4, 5, 7, 9, 12, 16, 17, 20, 22, 25, $27,28,32,33,39,41,44,45,49,50,56,60,64,76,78$, 79,$80 ; \alpha=.95$.

3. Factor III: Sensory Motor accounted for $22.4 \%$ of the variance. Questions: 2, 10, 19, 24, 29, 34, 35, 36, 40, $46,51,54,57,59,65,70,71,73 ; \alpha=.87$.

4. Factor IV: Social Anxiety accounted for $18.9 \%$ of the variance. Questions: 21, 23, 26, 30, 42, 47, 55, 61, 63, $67,69,75 ; \alpha=.89$.

A confirmatory factor analysis using these four factors showed that the coefficient of variation of the factor loadings was $20,18,18$, and $19 \%$, respectively, implying similar loadings for each item within a given factor. A comparison of factor loadings (differential item function) by gender and by ASD vs. non-ASD showed no significant differences with the mean difference of $18 \%$ or less.

\section{Test-Retest Reliability}

Fifteen ASD subjects and fifteen comparison subjects without another DSM-IV-TR diagnosis were retested at UCLA after a mean interval of 1 year (maximum interval 15 months, minimum interval 10 months) The initial RAADS-R mean score for the ASA subjects was 155 , and when repeated was 149 . The initial RAADS-R mean score for the comparison subjects was 20 , and 21 when repeated (Table 7 contains the statistical analyses for test-retest data).

\section{University/Center Effect}

The total mean RAADS-R scores for the ASD subjects from each of the 9 centers were significantly different from the comparison groups $(p<.0001)$ and there were significant differences between the universities (see Table 8).

\section{Clinical Implications of RAADS-R Scores}

A RAADS-R score of 65 or greater is consistent with a clinical diagnosis of ASD. A RAADS-R score of 64 or lower is not consistent with a diagnosis of ASD (sensitivity $=97 \%$, specificity $=100 \%$ ). It must be emphasized,

Table 7 Test-retest data statistical analysis

\begin{tabular}{lcll}
\hline Subject group & Number & Pearson $r=$ & Spearman Rho= \\
\hline All subjects & 30 & .987 & .956 \\
Autistic & 7 & 1 & 1 \\
Asperger & 8 & .939 & .952 \\
Comparisons & 15 & .874 & .764 \\
\hline
\end{tabular}

Table 8 RAADS-R scores and ranges by research center: ASD subjects

\begin{tabular}{lrlrl}
\hline Research center & $N$ & \multicolumn{3}{l}{ RAADS-R scores } \\
\cline { 3 - 5 } & & Mean 146 & Min & Max \\
\hline UCLA & 53 & 146 & 84 & 222 \\
Yale & 31 & 115 & 48 & 178 \\
Mount Sinai & 19 & 123 & 73 & 166 \\
University of Utah & 7 & 92 & 72 & 109 \\
Monsah University, Australia & 13 & 138 & 62 & 172 \\
Aspect, Sydney & 14 & 118 & 58 & 193 \\
Griffith University, Australia & 42 & 146 & 56 & 227 \\
Geneva Center, Canada & 8 & 148 & 107 & 216 \\
University of London & 14 & 135 & 67 & 205 \\
\hline
\end{tabular}

however, that if a subject has a score of 64 or lower but clinical judgment indicates that ASD is present, the clinical judgment should take precedence. This is due to the many limitations of self-rating scales that will be elaborated upon in the discussion section.

\section{Discussion}

The RAADS-R is a highly specific (100\%) and sensitive (97\%) instrument that is useful as an adjunct clinical diagnostic tool. Based on the results of this study and the RAADS pilot study, a RAADS-R score of 65 or higher is consistent with a diagnosis of ASD. The ASD subjects' scores for each question were significantly different from the comparison subjects'. Additionally, the mean RAADS-R scores of the ASD subjects and the comparison subjects were significantly different in each of the nine centers, as well as all of the centers combined. Both the clinically assigned question-domains and the statistically derived factors demonstrated high internal consistency. The RAADS-R had high test-retest reliability in a limited number of subjects. The RAADS-R was designed to assist clinicians in diagnosing adults $(18+)$ with suspected ASD. It is designed to be administered by clinicians in a clinical setting. It is not intended to be a mail in or an online screening instrument. The questions are designed for individuals with average IQ and above. This is a population with mild or subclinical ASD. These individuals often escape diagnosis. The RAADS-R was specifically designed to capture that population.

Limitations

Scales based on self-reports have inherent limitations: First, a subject may not understand a question, and while 
answering to the best of their ability, give a misleading answer. This was mitigated in the present study by having a clinician remain with the subject to clarify possible misunderstandings during the administration of the RAADS-R.

Second is the issue of unawareness i.e., "denial" of symptoms or over reporting of symptoms. To control for this we added the SRS-A, which is a second party instrument. The concordance rate was $95.95 \%$, which indicates good concurrent validity. However, since only 69 subjects were available to complete the SRS-A, further studies are warranted.

Six subjects whose clinical diagnoses and ADOS module 4 scores placed them in the ASD group, scored below the RAADS-R ASD cut off point of 65. Their mean age was 19.9 years. Each of these six "false negative" subjects was reported by their family members to be invested in appearing "as normal as they could be." The ANOVA results show a positive correlation with age in the ASD subjects with older subjects scoring higher (see Results section). Possible explanations are: (a) the younger subjects were denying symptoms, (b) the younger subjects received treatment since childhood and now believed they were "normal," and (c) three of the cases from Yale's sample have low functioning siblings with autism. These subjects may see themselves relatively unaffected for this reason. This stresses the importance for clinicians to search very carefully for symptoms when diagnosing young adults, given their propensity to want to "look as normal as possible."

Another confounding factor is that the same symptoms of ASD reported to be unobtrusive by some subjects may be serious to incapacitating in others. For instance, a young man reported that hypersensitivity to lights and sounds caused him to be "completely socially isolated," while another with the same symptoms told us, "I go to carnivals, circuses, and state fairs, every chance I get-I enjoy the flashing lights and sounds of the barkers and noises the merry go round makes." "And I stay as long as I can," he added with a smile.

\section{Factor Analysis}

As noted in the Results section, four factors emerged from Factor Analysis. The Cronbach alpha coefficients were satisfactory (social relatedness $=.93$, circumscribed interests $=.95$, sensory motor $=.87$, social anxiety $=.89$ ), indicating good internal consistency. For the clinical domain, the Cronbach alpha for language was the weakest. Factor IV contains questions relating to social anxiety and anxiety in general. This factor is important to examine. Targeting the social anxiety questions within the instrument may help the clinician differentiate social anxiety from ASD. The two diagnoses are often difficult to differentiate.
University Effect

As shown in the results section, there were significant differences of the mean RAADS-R scores among the nine research centers. This is most likely due to a large variation in the number of subjects per center (maximum 53 to a minimum of 7). Since the mean RAADS-R score for each center was significantly different from the mean RAADS-R scores of the comparison subjects $(p<.0001)$, it is likely that the differences among centers would diminish if those with fewer subjects were to expand their database. Further validation studies are indicated.

\section{Relevance to DSM-V}

Results show significant statistical differences between the autism and Asperger's subjects and comparison subjects with and without another DSM-IV-TR diagnosis. There were no significant differences between the autism and Asperger's groups, which supports the newly proposed DSM-V diagnostic criteria. Recently proposed DSM-V criteria combines the two groups, creating an Autism Spectrum Disorder diagnosis.

\section{Conclusions}

The RAADS-R proved to be highly accurate in discriminating between subjects with ASD and those without a DSM-IV-TR diagnosis and with another DSM-IV-TR diagnosis (sensitivity $=97 \%$, specificity $=100 \%$, testretest reliability $=.987$, accuracy $=98.5 \%$, ). No difficulties were reported in administration even though subjects were evaluated at nine medical centers on three continents. Mean RAADS-R scores were highly significantly different $(p<.0001)$ between ASD and comparison subjects at each of the nine centers, and all eighty questions were significantly different between ASD and comparison subjects. The fact that this was true even when the comparison cases had a variety of DSM-IV-TR diagnoses other than ASD makes the RAADS-R particularly useful in adult clinical settings.

Acknowledgments We wish to express our gratitude to professor Ami Klin for his mentorship and consultation, Avril Brereton, Latha Sooraya, Trevor Clark, Vicky Gibbs, Stephanie Moseser-Warren the Alan B. Slifka foundation, Marilyn Miller and Angela Pih, for their contributions and support.

Open Access This article is distributed under the terms of the Creative Commons Attribution Noncommercial License which permits any noncommercial use, distribution, and reproduction in any medium, provided the original author(s) and source are credited. 
Appendix 1: The Ritvo Autism Asperger's Diagnostic Scale-Revised (RAADS-R) and Scoring Instructions

\section{RAADS-R $\mathbb{C}$}

\section{Ritvo Autism-Asperger's Diagnostic Scale - Revised}

All information on this scale is strictly confidential

* 1. Your name

* 2. Your address

*3. Your phone number (

* 4. Today's date

* 5. Your age in years

Your gender:

Marital status:

Do you have children?

*6. Male

* 8. Single

* 11. Not married but in a significant relationship
* 7. Female

* 9. Married

*13. No

* 14 If yes, list their sexes, ages and any psychiatric or neurological disabilities they may have including autism and Asperger's Disorder:

a.

b.

c.

d.

e.

Do you have or have you ever had a driver's license? Yes $\square$ No $\square$

* 17. The highest grade passed or degree earned in school

* 18. Was this in regular class?

* 19. Or special education?

* 20. Have you ever been diagnosed or labelled as having Autistic Disorder, Asperger's Disorder, High Functioning Autism, Pervasive Developmental Disorder, Dyslexia, Mental Retardation, Learning Disability or another psychiatric or neurological disorder?

If so, please name the diagnosis or label, when it was given and by whom (name the doctor, clinic or a school). Please use other paper if necessary.

Diagnosis:

Name of doctor, clinic or other:

Date of diagnosis:

When did you begin speaking?

* 21. I began at the usual time (around my second birthday at 24 months of age)

* 22. I began speaking late (at or later than age $2 \frac{1}{2}$ or 30 months)

* 23. I have no information as to when I began speaking or early language problems.

It will take you about an hour to answer all the questions. 
Some life experiences and personality characteristics

that may apply to you

1* I am a sympathetic person.

2. I often use words and phrases from movies and television in conversations.

3. I am often surprised when others tell me I have been rude.

4. Sometimes I talk too loudly or too softly, and I am not aware of it.

5. I often don't know how to act in social situations.

6* I can "put myself in other people's shoes."

7. I have a hard time figuring out what some phrases mean, like "you are the apple of my eye."

8. I only like to talk to people who share my special interests.

9. I focus on details rather than the overall idea.

10. I always notice how food feels in my mouth. This is more important to me than how it tastes.

11* I miss my best friends or family when we are apart for a long time.

12. Sometimes I offend others by saying what I am thinking, even if I don't mean to.

13. I only like to think and talk about a few things that interest me.

14. I'd rather go out to eat in a restaurant by myself than with someone I know.

15. I cannot imagine what it would be like to be someone else.

16. I have been told that I am clumsy or uncoordinated.

17. Others consider me odd or different.

18* I understand when friends need to be comforted.

19. I am very sensitive to the way my clothes feel when I touch them. How they feel is more important to me than how they look.

\section{Check only one column}

\begin{tabular}{ccc}
$\begin{array}{c}\text { True now } \\
\text { and when I } \\
\text { was young }\end{array}$ & $\begin{array}{c}\text { True only } \\
\text { now }\end{array}$ & $\begin{array}{c}\text { True only } \\
\text { when I was } \\
\text { younger } \\
\text { than 16 }\end{array}$ \\
\hline
\end{tabular}
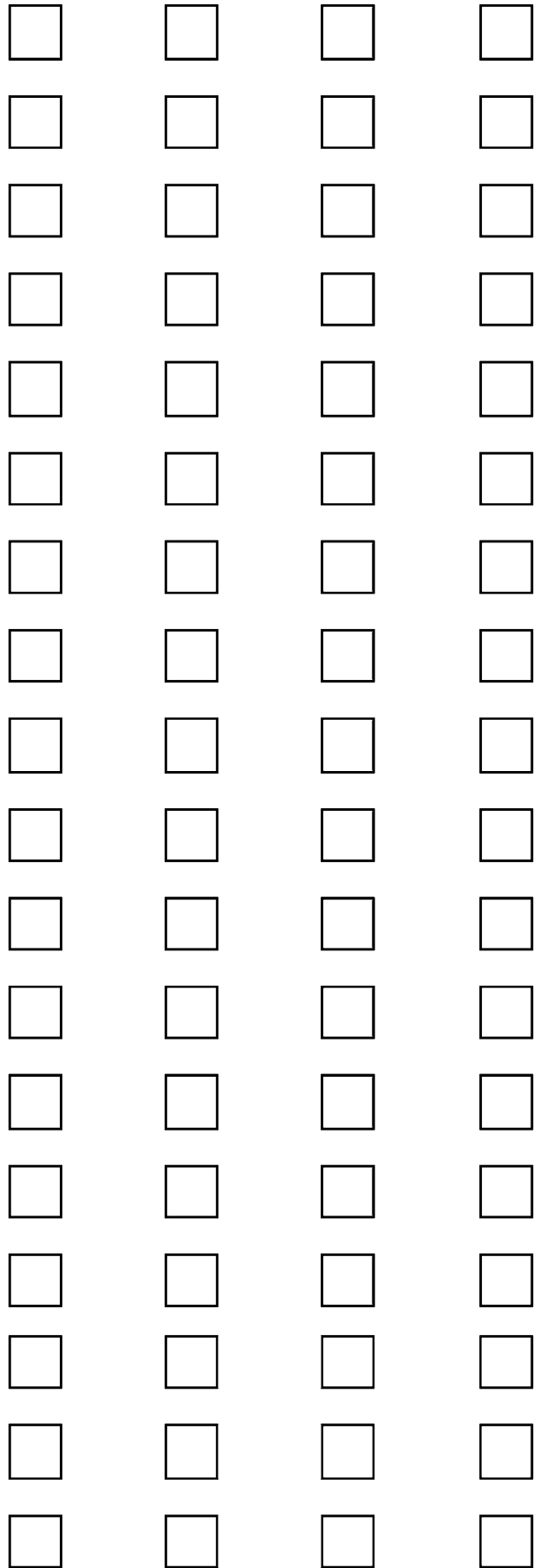

Please continue on the next page

(C) Riva Ariella Ritvo, Ph.D. and Edward Ritvo, M.D., 2007 
Some life experiences and personality characteristics

that may apply to you

20. I like to copy the way certain people speak and act. It helps me appear more normal.

21. It can be very intimidating for me to talk to more than one person at the same time.

22. I have to "act normal" to please other people and make them like me.

23* Meeting new people is usually easy for me.

24. I get highly confused when someone interrupts me when I am talking about something I am very interested in.

25. It is difficult for me to understand how other people are feeling when we are talking.

26* I like having a conversation with several people, for instance around a dinner table, at school or at work.

27. I take things too literally, so I often miss what people are trying to say.

28. It is very difficult for me to understand when someone is embarrassed or jealous.

29. Some ordinary textures that do not bother others feel very offensive when they touch my skin.

30. I get extremely upset when the way I like to do things is suddenly changed.

31. I have never wanted or needed to have what other people call an "intimate relationship."

32. It is difficult for me to start and stop a conversation. I need to keep going until I am finished.

33* I speak with a normal rhythm.

34. The same sound, color or texture can suddenly change from very sensitive to very dull.

35. The phrase "I've got you under my skin" makes me very uncomfortable.

36. Sometimes the sound of a word or a high-pitched noise can be painful to my ears.

37* I am an understanding type of person.

\section{Check only one column}

$\begin{array}{lccc}\begin{array}{c}\text { True now } \\ \text { and when I } \\ \text { was young }\end{array} & \begin{array}{c}\text { True only } \\ \text { now }\end{array} & \begin{array}{c}\text { True only } \\ \text { when I was } \\ \text { younger }\end{array} & \text { Never true } \\ & & \text { than I6 }\end{array}$

than 16
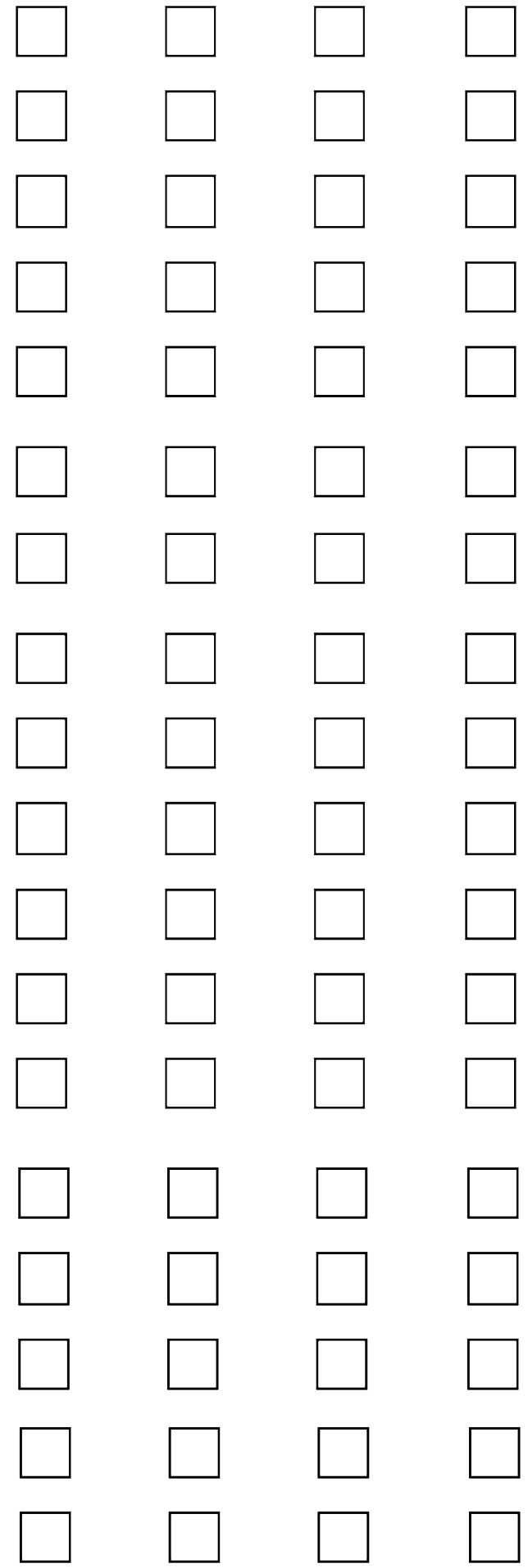
Some life experiences and personality characteristics that may apply to you

38. I do not connect with characters in movies and cannot feel what they feel.

39. I cannot tell when someone is flirting with me.

40. I can see in my mind in exact detail things that I am interested in.

41. I keep lists of things that interest me, even when they have no practical use (for example sports statistics, train schedules, calendar dates, historical facts and dates).

42. When I feel overwhelmed by my senses, I have to isolate myself to shut them down.

43* I like to talk things over with my friends.

44. I cannot tell if someone is interested or bored with what I am saying.

45. It can be very hard to read someone's face, hand and body movements when they are talking.

46. The same thing (like clothes or temperatures) can feel very different to me at different times.

47* I feel very comfortable with dating or being in social situations with others.

48* I try to be as helpful as I can when other people tell me their personal problems.

49. I have been told that I have an unusual voice (for example flat, monotone, childish, or highpitched).

50. Sometimes a thought or a subject gets stuck in my mind and I have to talk about it even if no one is interested.

51. I do certain things with my hands over and over again (like flapping, twirling sticks or strings, waving things by my eyes).

52. I have never been interested in what most of the people I know consider interesting.

53* I am considered a compassionate type of person.

54. I get along with other people by following a set of specific rules that help me look normal.

55. It is very difficult for me to work and function in groups.

\section{Check only one column}

True now and when I was young
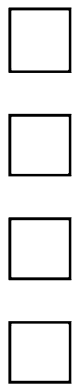

True only

now

True only when I was

younger

than 16
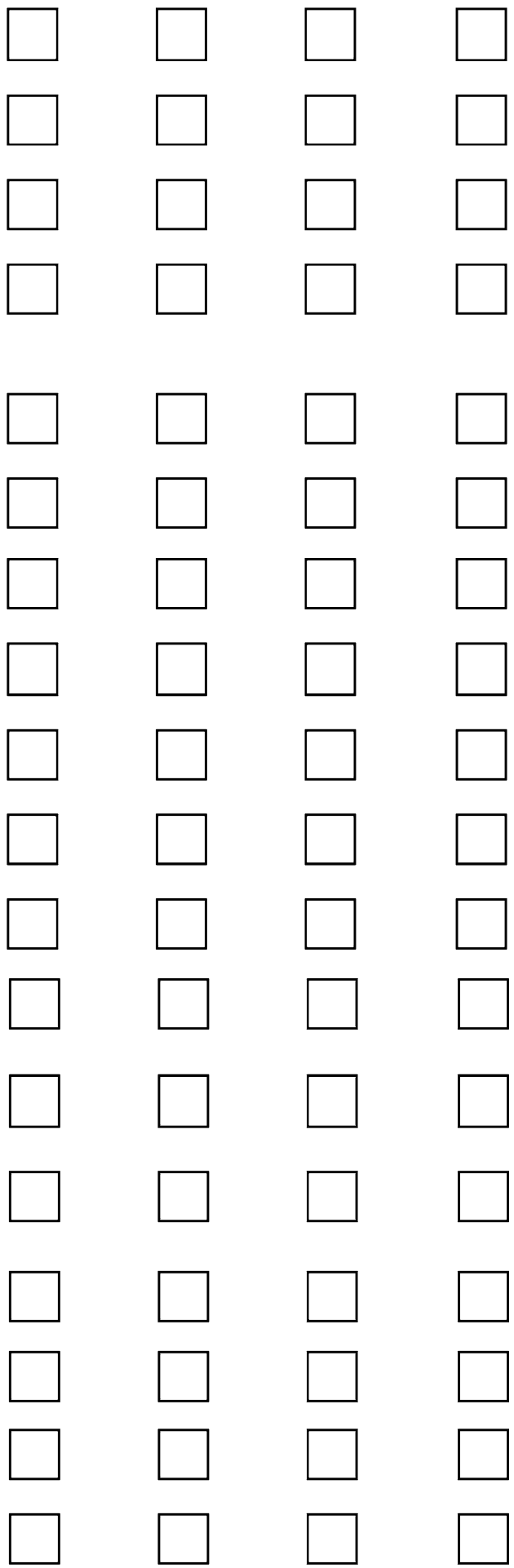

Please continue on the next page

(C) Riva Ariella Ritvo, Ph.D. and Edward Ritvo, M.D., 2007 
Some life experiences and personality characteristics

that may apply to you

56. When I am talking to someone, it is hard to change the subject. If the other person does so, I can get very upset and confused.

57. Sometimes I have to cover my ears to block out painful noises (like vacuum cleaners or people talking too much or too loudly).

58* I can chat and make small talk with people.

59. Sometimes things that should feel painful are not (for instance when I hurt myself or burn my hand on a stove).

60. When talking to someone, I have a hard time telling when it is my turn to talk or to listen.

61. I am considered a loner by those who know me best.

62* I usually speak in a normal tone.

63. I like things to be exactly the same day after day and even small changes in my routines upset me.

64. How to make friends and socialize is a mystery to me.

65. It calms me to spin around or to rock in a chair when I am feeling stressed.

66. The phrase, "He wears his heart on his sleeve," does not make sense to me.

67. If I am in a place where there are many smells, textures to feel, noises or bright lights, I feel anxious or frightened.

68* I can tell when someone says one thing but means something else.

69. I like to be by myself as much as I can.

70. I keep my thoughts stacked in my memory like they are on filing cards, and I pick out the ones I need by looking through the stack and finding the right one (or another unique way).

71. The same sound sometimes seems very loud or very soft, even though I know it has not changed.

72* I enjoy spending time eating and talking with my family and friends.

73. I can't tolerate things I dislike (like smells, textures, sounds or colors).

Check only one column

$\begin{array}{lccc}\begin{array}{c}\text { True now } \\ \text { and when I } \\ \text { was young }\end{array} & \begin{array}{c}\text { True only } \\ \text { now }\end{array} & \begin{array}{c}\text { True only } \\ \text { when I was } \\ \text { younger } \\ \text { than 16 }\end{array} & \text { Never true } \\ & & & \end{array}$
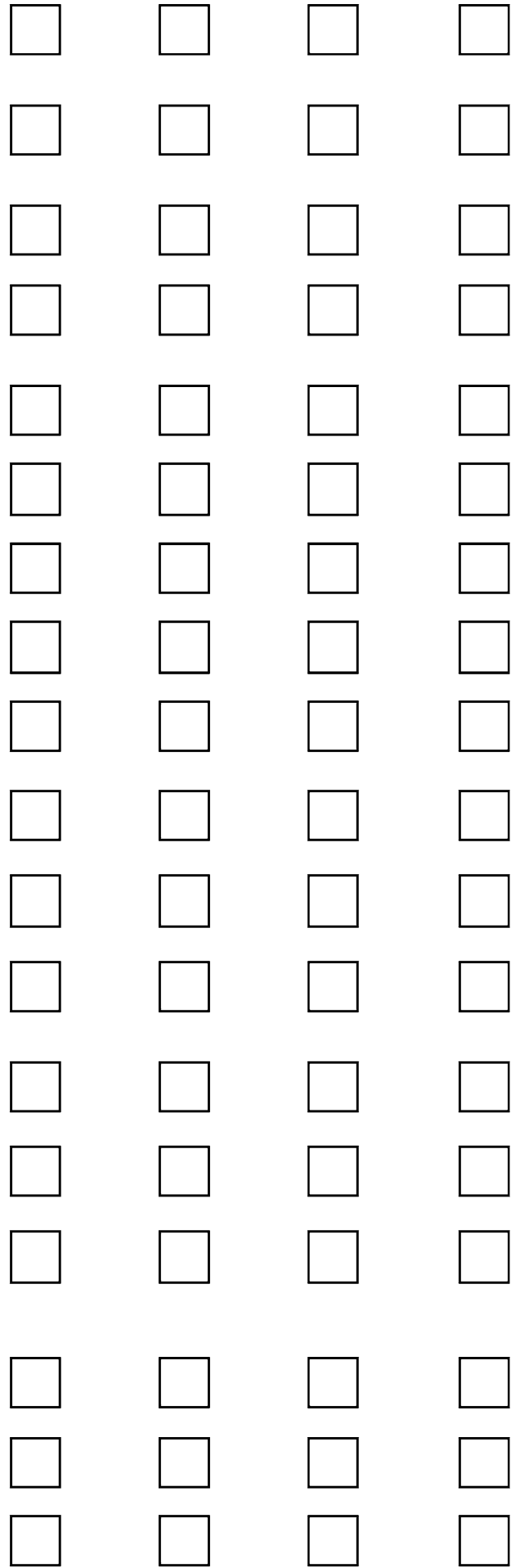
Some life experiences and personality characteristics

that may apply to you

74. I don't like to be hugged or held.

75. When I go somewhere, I have to follow a familiar route or I can get very confused and upset.

76. It is difficult to figure out what other people expect of me.

77* I like to have close friends.

78. People tell me that I give too much detail.

79. I am often told that I ask embarrassing questions.

80. I tend to point out other people's mistakes.

\section{Check only one column}

$\begin{array}{cccc}\begin{array}{c}\text { True now } \\ \text { and when I } \\ \text { was young }\end{array} & \begin{array}{c}\text { True only } \\ \text { now }\end{array} & \begin{array}{c}\text { True only } \\ \text { when I was } \\ \text { younger } \\ \text { than 16 }\end{array} & \text { Never true } \\ & & & \end{array}$
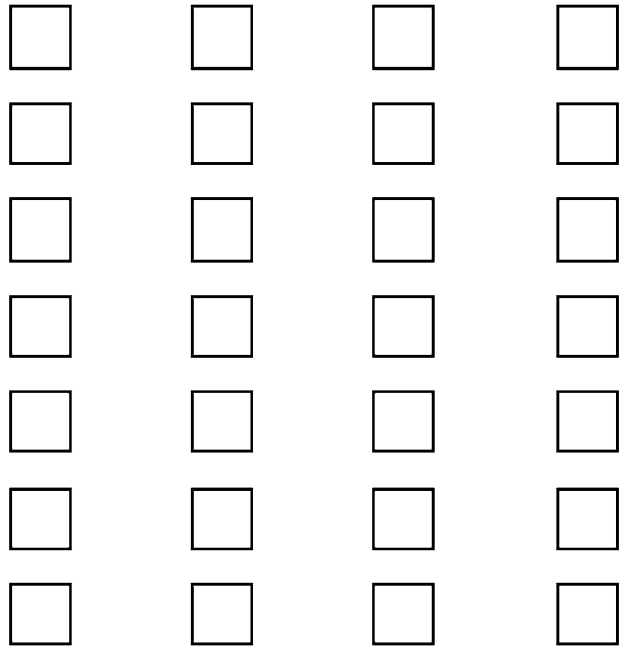

Thank you for your cooperation!

(C) Riva Ariella Ritvo, Ph.D. and Edward Ritvo, M.D., 2007

\section{Appendix 2: Instructions for Scoring the Scale}

The scale contains two types of questions:

1. 64 questions describing specific symptoms of ASD. These are scored in order of severity from: "true now and when I was young" $=3$, to "never true" $=0$.
2. 16 questions describing non symptomatic (normative) behaviors. These are score: "true now and when I was young" $=0$ to "never true" $=3$. These questions have asterisks by their number (Table 9).

Table 9 Scores for the four possible answers

\begin{tabular}{|c|c|c|c|c|}
\hline Answer checked by the subject & $\begin{array}{l}\text { True now and } \\
\text { when I was young }\end{array}$ & Only true now & True only when i was young & Never true \\
\hline $\begin{array}{l}\text { Symptom based questions } \\
N=64 \\
\text { Example: "I take things too literally } \\
\text { so I often miss what } \\
\text { people are trying to say". } \\
\text { (No asterisk after question number) }\end{array}$ & 3 & 2 & 1 & 0 \\
\hline $\begin{array}{l}\text { Normative questions } \\
N=16\end{array}$ & 0 & 1 & 2 & 3 \\
\hline $\begin{array}{l}\text { Example: "I" * "I am a sympathetic person". } \\
\text { (Asterisk after question number) }\end{array}$ & & & & \\
\hline
\end{tabular}




\section{References}

American Psychiatric Association. (2000). Diagnostic and statistical manual of mental disorders (4th ed.). Washington, DC: American Psychiatric Association.

APA DSM-5. (2010). Retrieved June 9, 2010, from http://www. dsm5.org/Pages/Default.aspx.

Attwood, T. (1998). Asperger's syndrome, a guide for parents and professionals. London, England: Jessica Kingsley Publications.

Baron-Cohen, S., Wheelwright, S., Robinson, J., \& Woodbury-Smith, M. (2005). The adult Asperger's assessment (AAA): A diagnostic method. Journal of Autism and Developmental Disorders, 35(6), 807-819.

Baron-Cohen, S., Wheelwright, S., Skinner, R., Martin, J., \& Clubley, E. (2001). The autism-spectrum quotient (AQ): Evidence from Asperger Syndrome/high-functioning autism, males and females, scientists and mathematicians. Journal of Autism and Developmental Disorders, 31(6), 5-17.

Bolte, S., Postka, F., \& Constantino, J. (2008). Assessing autistic traits: Cross- cultural validation of the social responsiveness scale (SRS). Autism Research, 1(6), 354-363.

Ehlers, S., Gillberg, C., \& Wing, L. (1999). A screening questionnaire for Asperger syndrome and other high-functioning autism spectrum disorders in school age children. Journal of Autism and Developmental Disorders, 29(2), 129-141.

Fitzgerald, M., \& Corvin, A. (2001). Diagnosis and differential diagnosis of Asperger syndrome. Advances in Psychiatric Treatment, 7, 310-318.

Klin, A., Pauls, D., Schultz, R., \& Volkmar, F. (2005). Three diagnostic approaches to Asperger's syndrome: Implications for research. Journal of Autism and Developmental Disorders, 35(2), 221-234.

Ritvo, R., Ritvo, E., Guthrie, D., Yuwiler, A., Ritvo, M., \& Weisbender, L. (2008). A scale to assist the diagnosis of Autism and Asperger's disorder in Adults (RAADS): A pilot study. Journal of Autism and Developmental Disorders, 38(2), 213-223.

Volkmar, F., \& Lord, C. (1998). Diagnosis and definition of autism and other pervasive developmental disorders. In F. Volkmar (Ed.), Autism and pervasive developmental disorders (pp. 1-31). Cambridge, England: Cambridge University Press.

World Health Organization. (1992). International classification of diseases: Diagnostic criteria for research (10th ed.). Geneva: World Health Organization. 